\title{
A Case of a Mucinous Adenocarcinoma Arising from a Rectal Diverticulum
}

\author{
Jang Hoon Kwon, Koon Hee Han, Woo-Sung Chang, Ki-Ho Nam, Myoung Sik Han ${ }^{1}$, Jae Hong Ahn², \\ Sang Hak $\mathrm{Han}^{3}$, Gab Jin Cheon \\ Departments of Internal Medicine, ${ }^{1}$ General Surgery, and ${ }^{2}$ Diagnostic Radiology, Gangneung Asan Hospital, University of Ulsan College of \\ Medicine, Gangneung; ${ }^{3}$ Department of Pathology, Hallym University Chuncheon Sacred Heart Hospital, Chuncheon, Korea
}

The occurrence of an adenocarcinoma arising from a rectal diverticulum that causes mechanical ileus is very rare. Recently, we diagnosed a case of a mucinous adenocarcinoma in a rectal diverticulum after an emergent abdominal perineal resection and permanent colostomy by laparotomy. Here, we present a case report and a review of the literature.

Keywords: Mucinous adenocarcinoma; Diverticulum; Ileus

\section{INTRODUCTION}

Diverticular disease primarily involves the sigmoid colon, followed by the ascending colon and cecum. Rectal involvement is extremely rare, with only a few reports in the literature since 1911 [1]. Most rectal diverticula do not require treatment because many cases are asymptomatic. Surgical intervention is necessary when the patient suffers from a diverticular complication [2]. In this case, an emergent laparotomy following an abdominal perineal resection (APR) and permanent colostomy was performed because of an impending bowel perforation caused by a mucinous adenocarcinoma within the rectal diverticulum.

\section{CASE REPORT}

A 77-year-old man with a 10-day history of chronic constipation and abdominal distension was seen at the emergency room. The patient's medical history included right hemiparesis due to left

Received: January 4, 2012 - Accepted: June 10, 2012

Correspondence to: Koon Hee Han, M.D.

Department of Internal Medicine, Digestive Disease Center, Gangneung Asan Hospital, University of Ulsan College of Medicine, 38 Bangdong-gil, Gangneung 210-711, Korea

Tel: +82-33-610-4951, Fax: +82-33-641-4960

E-mail: hankoonhee@hanmail.net

(c) 2012 The Korean Society of Coloproctology

This is an open-access article distributed under the terms of the Creative Commons Attribution NonCommercial License (http://creativecommons.org/licenses/by-nc/3.0) which permits unrestricted noncommercial use, distribution, and reproduction in any medium, provided the original work is properly cited. basal ganglia infarction 8 years prior and systemic hypertension. On physical examination, he had abdominal distension with mild tenderness; however, he did not have rebound tenderness and his bowel sounds were increased. On digital rectal examination, there was an edematous soft mass-like lesion at a one-finger depth from the anal verge which was not tinged with blood. Laboratory studies revealed that the white blood cell count, hemoglobin, electrolytes, and liver function tests were within the normal range. The level of carcinoembryonal antigen was $17.0 \mathrm{ng} / \mathrm{mL}$ (normal range, 0 to $6 \mathrm{ng} / \mathrm{mL}$ ), and that of carbohydrate antigen $19-9$ was $99.3 \mathrm{U} /$ $\mathrm{mL}$ (normal range, 0 to $37 \mathrm{U} / \mathrm{mL}$ ). Plain abdominal radiography revealed a mechanical ileus with severe bowel distension (Fig. 1). A contrast enhanced computed tomography scan was suggestive of rectal cancer with a diffuse wall thickening that was $7 \mathrm{~cm}$ in length and was located $5 \mathrm{~cm}$ above the anal verge. The small bowel and colon were dilated with fecal retention due to a mechanical obstruction proximal to the mass lesion (Fig. 2). Colonoscopic findings revealed that the extruding mass was covered with normal mucosa (Fig. 3). Endoscopic biopsy showed only an inflammatory change; however, we clinically suspected rectal cancer, so additional pelvic magnetic resonance imaging (MRI) was performed. The pelvic MRI revealed a submucosal infiltrating enhanced lesion involving the rectum and containing round and tubular cystic lesions with perirectal fat infiltration (Fig. 4). A positron emission tomography scan showed rectal cancer because of increased fluorine-18 2-fluoro-2-deoxy-D-glucose uptake (max standardized uptake value, 7.7).

Because of negative biopsy results and radiologic findings that showed a submucosal infiltrating enhanced lesion, we suspected 


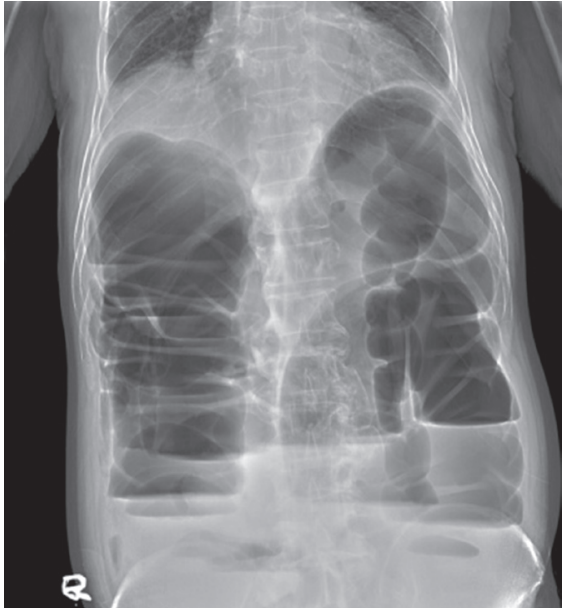

Fig. 1. Plain abdominal radiograph showing the colonic obstruction.

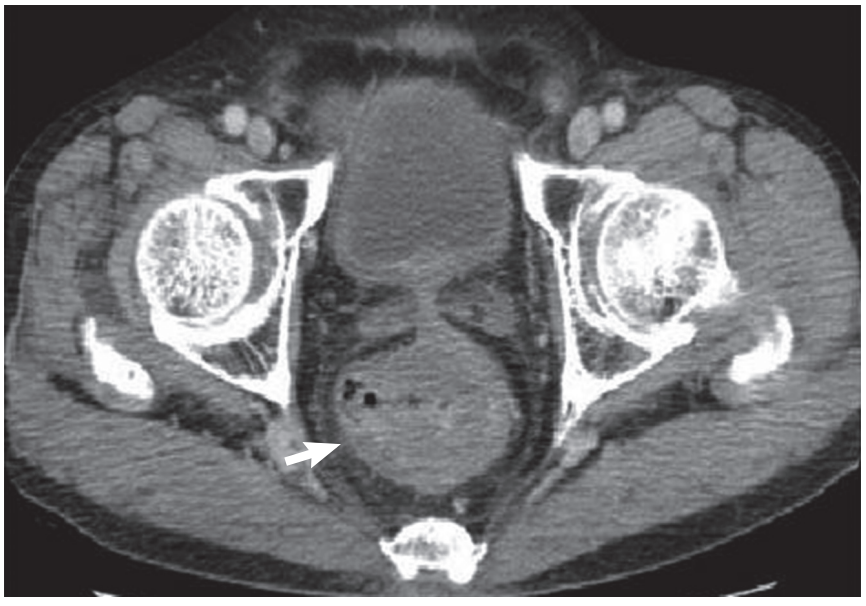

Fig. 2. Axial contrast-enhanced computed tomography scans showing circumferential thickening of the rectum (arrow).

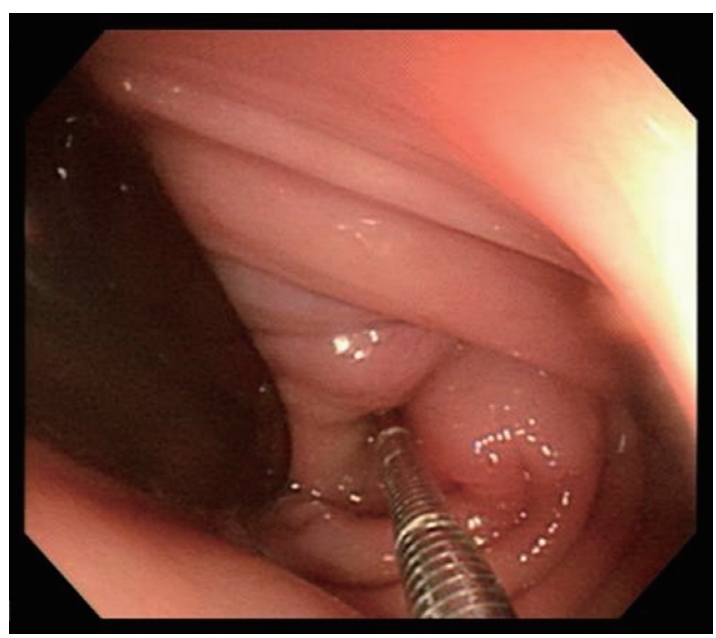

Fig. 3. Colonoscopic findings showing the extruding lesion. The biopsy revealed a normal mucosa epithelium. that the tumor was most likely a primary rectal linitis plastica and recommended surgical treatment for the patient. However, the patient declined surgery without pathologic confirmation and chose close observation and symptomatic treatment. One month later, he returned to the emergency room with recurrent abdominal distension and severe constipation. An emergent APR and permanent colostomy was performed due to signs of impending bowel perforation. During surgery which lasted for 5 hours, there were no special events, but pelvic dissection was difficult due to the infiltrative tumor mass and neighboring fibrosis. After surgery, the patient recovered without complications. Pathologic examination of the resected tissue revealed a mucinous adenocarcinoma in the rectal diverticulum involving the rectum $\left(4.2 \times 2 \times 1.5 \mathrm{~cm}^{3}\right)$ with extension to perirectal soft tissue; however, no lymphovascular perineural invasion or distant metastasis was detected (Fig. 5).

The cancer was located within a diverticulum, but the entrance of the diverticulum was not seen during endoscopy (Fig. 6). The negative endoscopic biopsy result was due to the fact that the mucous membrane was unaffected by the adenocarcinoma within the rectal diverticulum. After surgery, the patient received adju-

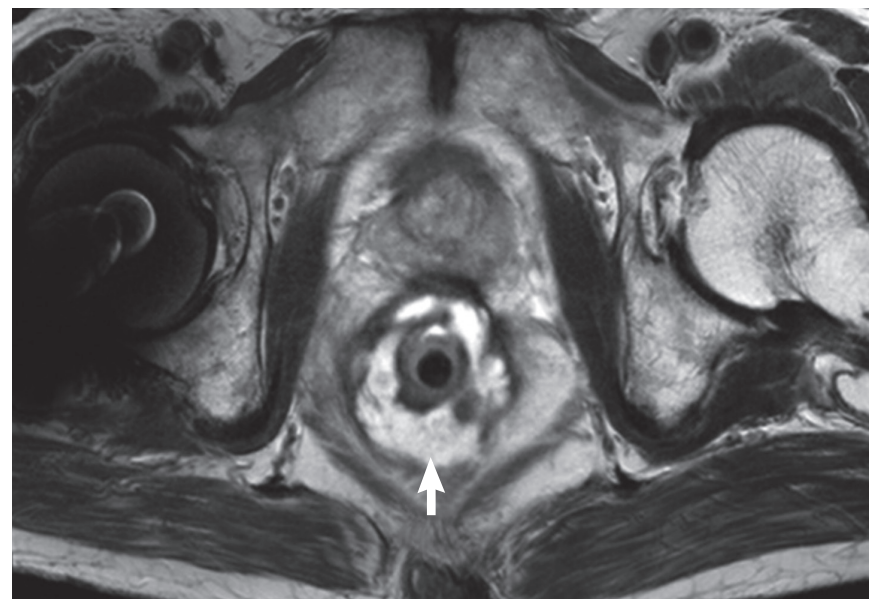

Fig. 4. Axial T2-weighted image showing the thickening of the rectum containing an intramural cystic lesion (arrow). The rectal tube is also seen.

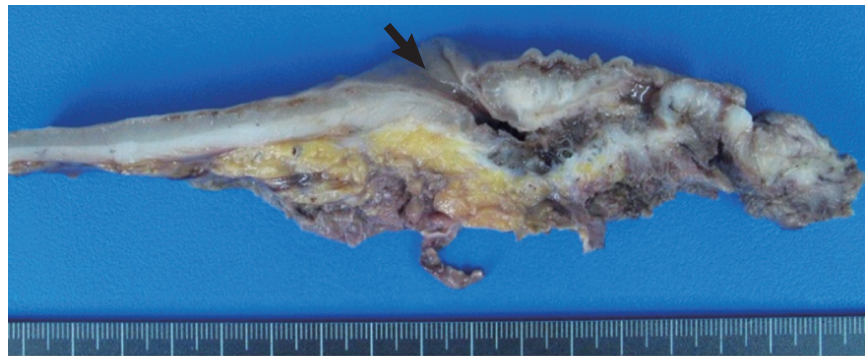

Fig. 5. Resected rectum showing the rectal diverticulum and the mucous material in the submucosal layer. The entrance of the rectal diverticulum was dilated by formalin fixation (arrow). 


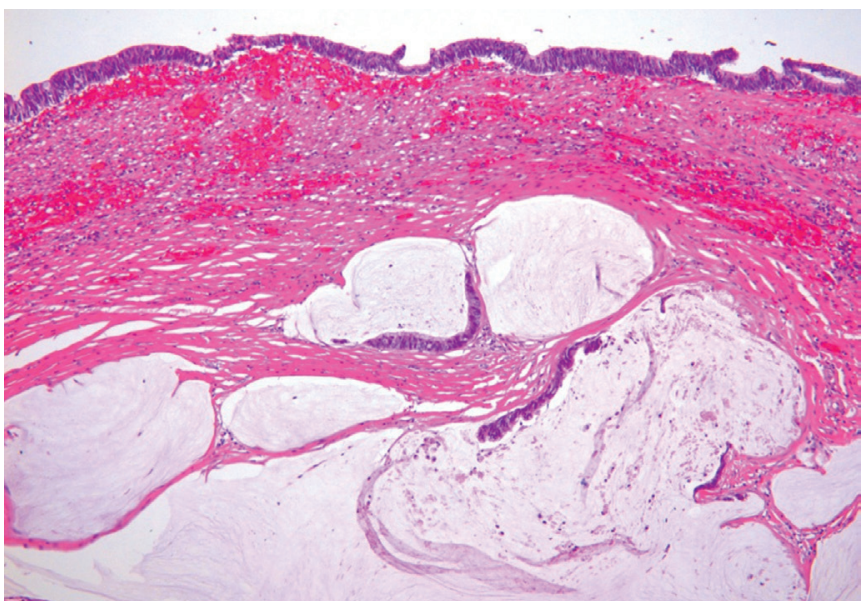

Fig. 6. Microscopic findings: the mucinous adenocarcinoma in the rectal diverticulum. More than $50 \%$ of the lesion is composed of a mucin-containing malignant epithelium $(\mathrm{H} \& \mathrm{E}, \times 100)$.

vant radiation therapy, and no recurrence has been detected for 12 months.

\section{DISCUSSION}

Diverticular disease primarily involves the sigmoid colon, accounting for $65 \%$ of the cases. Involvement of the entire colon occurs in up to $10 \%$ of the cases, with between 2 to $10 \%$ being limited to the transverse or ascending colon. However, rectal involvement is extremely rare. Two theories for the low incidence of rectal diverticula have been described. First, the muscle fibers of the taenia coli spread outward, thus surrounding the rectum and protecting it against intraluminal pressures [3]. Secondly, less constant internal pressure is exerted on the rectum by accumulated feces and by a lower peristaltic activity as compared with the sigmoid colon [4]. The causes of rectal diverticula remain unknown; possible predisposing factors include congenital anomalies (e.g., weakness in the circumferential muscle that surrounds the rectum), primary muscle atrophy, or the absence of supporting structures (e.g., the coccyx). In this case, such predisposing factors were not observed.

Most rectal diverticula do not require treatment because many cases are asymptomatic. Most patients that are symptomatic have a concurrent rectal diverticulum, as in the present case. Rectal diverticula may be inflamed with impacted feces and can progress to abscess formation and perforation. Other reported complications include rectal strictures, fistula development, and rarely rectal prolapse because of a large inverted diverticulum. Surgical intervention is necessary only in severe cases [5].

Although colonic diverticulosis and cancer are common diseases in patients over 60 years of age, cancer arising within a diverticulum is rare [6]. The possibility of a casual relationship between diverticulitis and cancer of the colon has been suggested. Some investigators have attempted to show an increased incidence of co- lonic carcinomas in patients with diverticulitis, suggesting that chronic inflammation leads to metaplasia and neoplastic changes [7]. However, others have suggested that no cause-effect relationship exists between an adenocarcinoma and diverticular disease of the colon based on the following evidence: 1) the rarity of adenocarcinomas arising within the diverticular mucosa, 2) the absence of occasional dysplasia in the diverticular mucosa, and 3) the often documented occurrence of adenocarcinomas in areas with no diverticula [8].

To the best of our knowledge, only one case of an adenocarcinoma in a rectal diverticulum has been reported in Japan [9]. This report was the first case of a symptomatic mucinous adenocarcinoma within a rectal diverticulum, which caused a mechanical obstruction. During colonoscopic examination, the rectal diverticulum was not visible because the orifice of the diverticulum was narrow and collapsed. In this case, the diagnosis was difficult because radiographic features and endoscopic findings of the lesion were similar to those of a primary rectal linitis plastica; however, the cancer was diagnosed at a relatively early stage because the mucinous adenocarcinoma secreted a thick mucinous material into the submucosal layer, which caused a colonic obstruction before perforating the diverticular wall.

\section{CONFLICT OF INTEREST}

No potential conflict of interest relevant to this article was reported.

\section{REFERENCES}

1. Jung SH, Kim JH. A case of solitary rectal diverticulum presenting with a retrorectal mass. Gut Liver 2010;4:394-7.

2. Piercy KT, Timaran C, Akin H. Rectal diverticula: report of a case and review of the literature. Dis Colon Rectum 2002;45:1116-7.

3. Giffin HZ. VII. Diverticulitis of the rectum: a report of two cases operated upon, one of them with carcinomatous degeneration. Ann Surg 1911;53:533-7.

4. Hadi NI, Shakoor KA, Waseem B. Is mucin content a prognostic indicator in colorectal carcinoma? J Surg Pak 2009;14:7-10.

5. Symonds DA, Vickery AL. Mucinous carcinoma of the colon and rectum. Cancer 1976;37:1891-900.

6. Cohn KH, Weimar JA, Fani K, DeSoto-LaPaix F. Adenocarcinoma arising within a colonic diverticulum: report of two cases and review of the literature. Surgery 1993;113:223-6.

7. Kikuchi T, Kotanagi H, Kon H, Koyama K, Ito S, Otaka M. Mucosal carcinoma within a colonic diverticulum. J Gastroenterol 1999;34:622-5.

8. Hines JR, Gordon RT. Adenocarcinoma arising in a diverticular abscess of the colon: report of a case. Dis Colon Rectum 1975;18: 49-51.

9. Yuri S, Satoshi M, Takashi S, Katsuhiko S, Hiroshi N. Cancer in rectal diverticulum with periproctal abscess caused by penetration of cancer. Jpn J Gastroenterol Surg 2007;40:233-8. 